\title{
An optimal class of non-degenerate potentials for second-order ordinary differential equations
}

Kaiming Shen and Meirong Zhang*

\section{"Correspondence:}

mzhang@math.tsinghua.edu.cn Department of Mathematical Sciences, Tsinghua University, Beijing, 100084, People's Republic of China

\begin{abstract}
By considering the Dirichlet boundary condition $x(0)=x(1)=0$, we say that $q \in L^{1}[0,1]$ is a non-degenerate potential if the ordinary differential equation $x^{\prime \prime}+q(t) x=0$ has only the trivial solution $x(t) \equiv 0$ which verifies the boundary condition. Starting with a non-degenerate positive constant potential $B$, in this paper, we will apply the Pontryagin maximum principle (PMP) in optimal control theory to find the optimal bound $r=r(A, B)$ for any $A \in[-\infty, B)$ such that any potential $q \in L^{1}[0,1]$ satisfying $A \leq q \leq B$ and $\int_{[0,1]} q(t) d t>r(A, B)$ is necessarily non-degenerate. Such a non-degeneracy problem can be considered as the dual problem in a series of papers by Li et al.
\end{abstract}

Keywords: non-degenerate potential; eigenvalue; optimal control; Pontryagin maximum principle; boundary value problem

\section{Introduction}

Let us introduce the notion of non-degenerate potentials by considering the Dirichlet boundary condition.

Definition 1 Let $q \in L^{1}[0,1]$ be an integrable potential. If the following problem

$$
\begin{aligned}
& x^{\prime \prime}+q(t) x=0, \quad t \in[0,1], \\
& x(0)=x(1)=0,
\end{aligned}
$$

has only the trivial solution $x \equiv 0$, we say that $q(t)$ is a non-degenerate potential, or problem (1)-(2) is non-degenerate.

If problem (1)-(2) is non-degenerate, then, for any $h \in L^{1}[0,1]$, by the Fredholm alternative principle, the inhomogeneous equation

$$
x^{\prime \prime}+q(t) x=h(t), \quad t \in[0,1],
$$

has a unique solution $x(t)$ verifying boundary condition (2). Thus one can also say that problem (1)-(2) is non-resonant or invertible, as seen from different literature sources.

(c) 2015 Shen and Zhang. This article is distributed under the terms of the Creative Commons Attribution 4.0 International License (http://creativecommons.org/licenses/by/4.0/), which permits unrestricted use, distribution, and reproduction in any medium, provided you give appropriate credit to the original author(s) and the source, provide a link to the Creative Commons license, and indicate if changes were made. 
Non-degenerate potentials are very important in many problems. For example, it is well known that non-degenerate potentials play an important role in the solvability of boundary value problems of semilinear differential equations [1-8]. The maximum and antimaximum principles for various boundary value problems are also related with nondegenerate potentials [9-12]. More importantly, in recent years, it was found that the solvability, the exact multiplicity and the stability of solutions of several interesting classes of superlinear differential equations of the Landesman-Lazer type or of the AmbrosettiProdi type can be obtained from the characterization of non-degenerate potentials $[1,2$, 13-16].

All of these applications are based on explicit construction of non-degenerate potentials. To describe some known classes of non-degenerate potentials, let us use

$$
\lambda_{1}(q)<\lambda_{2}(q)<\cdots<\lambda_{m}(q)<\cdots
$$

to denote all eigenvalues of

$$
\ddot{x}+(\lambda+q(t)) x=0
$$

with boundary condition (2). Then the non-degeneracy can be explained using eigenvalues as follows.

Lemma 2 For $q \in L^{1}[0,1]$, problem (1)-(2) is non-degenerate if and only if $\lambda_{m}(q) \neq 0$ for all $m \in \mathbf{N}$.

Throughout this paper, denote

$$
\mu_{m}:=\lambda_{m}(0)=(m \pi)^{2}, \quad m \in \mathbf{N},
$$

the eigenvalues of problem (3)-(2) with zero potential $q=0$. Then a constant potential $q=$ $c \in \mathbf{R}$ is non-degenerate if and only if $c \neq \mu_{m}$ for all $m \in \mathbf{N}$. Moreover, by the comparison results for eigenvalues, it is well known that if $q$ satisfies

$$
\mu_{n-1} \prec q \prec \mu_{n}
$$

for some $n \in \mathbf{N}$, then $q$ is non-degenerate. This is a trivial, but frequently used, class of nondegenerate potentials. Here $\mu_{0}$ is understood as $-\infty$, and $q_{1} \prec q_{2}$ means that $q_{1}(t) \leq q_{2}(t)$ for all $t \in[0,1]$ and $q_{1}(t)<q_{2}(t)$ on some subset of $[0,1]$ of positive measure.

Because of Lemma 2, it is not an easy task to explicitly characterize non-trivial classes of non-degenerate potentials. However, in the study of semilinear ordinary differential equations, $\mathrm{Li}$ and his collaborators have applied the Pontryagin maximum principle (PMP) in the optimal control theory to construct several useful classes of non-degenerate potentials of (1) with respect to various boundary conditions like the Dirichlet, the Neumann, and the periodic boundary conditions. See $[3,4,6,7]$. Their main results, by taking the Dirichlet boundary condition as an example, are as follows. For $-\infty \leq A<B \leq+\infty$, denote

$$
\Omega_{A, B}:=\left\{q \in L^{1}[0,1]: A \leq q(t) \leq B \text { a.e. } t \in[0,1]\right\} .
$$


Starting with a non-degenerate constant potential $q \equiv A \in\left(\mu_{n}, \mu_{n+1}\right)$ for some $n \in \mathbf{N}$, for any $B \in\left[\mu_{n+1},+\infty\right)$, the optimal bound $R(A, B)$ has been found so that any $q \in \Omega_{A, B}$ satisfying

$$
\int_{0}^{1} q(t) d t<R(A, B)
$$

is a non-degenerate potential of problem (1)-(2). In fact, the bound $R:=R(A, B) \in\left(A, \mu_{n+1}\right]$ is uniquely determined by the following equation:

$$
\frac{1}{\sqrt{B}} \cot \frac{\sqrt{B}}{2 n} \frac{R-A}{B-A}=\frac{1}{\sqrt{A}} \tan \frac{\sqrt{A}}{2 n} \frac{B-R}{B-A} .
$$

Moreover, by letting

$$
R(A,+\infty):=\lim _{B \rightarrow+\infty} R(A, B)=A+2 n \tan \frac{\sqrt{A}}{2 n}
$$

$R(A,+\infty)$ is the corresponding optimal bound so that potentials $q \in \Omega_{A,+\infty}$ satisfying

$$
\int_{0}^{1} q(t) d t<R(A,+\infty)
$$

are also non-degenerate.

In [17], the second author of this paper has used some Sobolev inequalities to characterize another important class of non-constant non-degenerate potentials for problem (1)-(2) and its $p$-Laplacian counterpart. This class of non-degenerate potentials is used in $[9,10]$. It is worth mentioning that papers $[18-20]$ on the Lyapunov-type inequalities are also related with non-degenerate potentials.

In this paper, we study the following problem which can be considered as a dual problem to that in $[3,4,6,7]$. Given an upper bound

$$
B \in\left(\mu_{n}, \mu_{n+1}\right) \quad \text { for some } n \in \mathbf{N} \text {, i.e., } n=[\sqrt{B} / \pi] \in \mathbf{N} \text {. }
$$

In case $A \in\left(\mu_{n}, B\right)$, any $q \in \Omega_{A, B}$ is always non-degenerate. See condition (4). In the following we assume that

$$
A \in\left[-\infty, \mu_{n}\right]
$$

and consider potentials in $\Omega_{A, B}$. We aim at finding the optimal value $r=r(A, B)$ such that any $q \in \Omega_{A, B}$ satisfying

$$
\int_{0}^{1} q(t) d t>r(A, B)
$$

is necessarily non-degenerate. The final results are as follows.

Theorem 3 Let $B$ and $A$ be as in (6) and (7). 
- For $A \in\left(0, \mu_{n}\right]$, let

$$
r:=r(A, B) \in\left[\mu_{n}, A+n \pi(B-A) / \sqrt{B}\right] \subset\left[\mu_{n}, B\right)
$$

be the unique solution of the following equation:

$$
\sqrt{B} \cot \frac{\sqrt{B}}{2 n} \frac{r-A}{B-A}=\sqrt{A} \tan \frac{\sqrt{A}}{2 n} \frac{B-r}{B-A} .
$$

- For $A \in[-\infty, 0]$, let

$$
r(A, B):=n \pi \sqrt{B}=\pi \sqrt{B}[\sqrt{B} / \pi] .
$$

Then, for any $q \in \Omega_{A, B}$ satisfying condition (8), we have

$$
\lambda_{n}(q)<0<\lambda_{n+1}(q),
$$

which implies that problem (1)-(2) is non-degenerate. Moreover, the bound $r(A, B)$ is optimal in the sense that there exists $\hat{q} \in \Omega_{A, B}$ such that

$$
\int_{0}^{1} \hat{q}(t) d t=r(A, B)
$$

and $\hat{q}$ is degenerate.

One may compare equation (10) with (5). The results of Theorem 3 are obtained mainly using the PMP. However, different from the arguments in [3, 4, 6, 7], we will extensively apply the eigenvalue theory for problem (3). In fact, the non-degeneracy of potentials is a consequence of the estimates (12) on eigenvalues which are also optimal in a certain sense. Moreover, in order to prove the existence of the optimal control potentials for the optimal control problems deduced from the non-degenerate potential problems, we find that the (strong) continuous dependence of solutions and eigenvalues of (3) in potentials (with weak topology) in [21] can simplify the arguments significantly. In these senses, the present paper has given some simpler approach to the non-degeneracy problem.

The paper is organized as follows. At first, we introduce the Pontryagin maximum principle in optimal control theory and establish the connection between non-degeneracy problems and optimal control problems. Secondly, Theorem 3 is proved by solving equations in the PMP. Finally, we briefly consider non-degenerate potentials of (1) with the Neumann boundary condition and point out that the class of potentials in Theorem 3 is also non-degenerate with respect to the Neumann problem. As seen from applications of non-degenerate potentials to nonlinear differential equations mentioned above, it can be expected that the new class of non-degenerate potentials in Theorem 3 can lead to interesting applications to semilinear and superlinear differential equations.

\section{Control systems and the Pontryagin maximum principle}

For our purpose, let us introduce the following class of optimal control problems [22]. A control system consists of four elements: state-control trajectory, final state, set of admissible controls and cost functional. 
- the state-control trajectory $(\mathbf{x}, \mathbf{u})$ is characterized by a first-order ordinary differential system

$$
\dot{\mathbf{x}}=\mathbf{f}(\mathbf{x}, \mathbf{u}(t), t), \quad t \in\left[t_{0}, t_{f}\right]
$$

with a known initial state

$$
\mathbf{x}\left(t_{0}\right)=\mathbf{x}_{0}
$$

where $\mathbf{x}, \mathbf{x}_{0} \in \mathbf{R}^{n}, \mathbf{u} \in \mathbf{R}^{m}$;

- the final state is usually described by

$$
\mathbf{g}\left(\mathbf{x}\left(t_{f}\right)\right)=0
$$

where $\mathbf{g}: \mathbf{R}^{n} \rightarrow \mathbf{R}^{k}$ is a known function;

- the set of admissible controls is described by

$$
\begin{aligned}
\mathbf{U}_{\left[t_{0}, t_{f}\right]}:= & \left\{\mathbf{u}(t): \mathbf{u}(\cdot) \text { is a piecewise continuous function on }\left[t_{0}, t_{f}\right]\right. \\
& \text { such that } \mathbf{u}(t) \in U_{m} \text { for all } t \in\left[t_{0}, t_{f}\right] \text { and } \\
& \text { problem (13)-(14)-(15) has solutions } \mathbf{x}(t)\}
\end{aligned}
$$

where $U_{m} \subset \mathbf{R}^{m}$ is a known domain; and

- the cost functional is a functional of $\mathbf{u}(\cdot) \in \mathbf{U}_{\left[t_{0}, t_{f}\right]}$ taking the following form:

$$
J[\mathbf{u}(\cdot)]=\int_{t_{0}}^{t_{f}} L(\mathbf{x}(t), \mathbf{u}(t), t) d t
$$

where $L(\mathbf{x}, \mathbf{u}, t)$ is a known function.

The optimal control problem is to find an admissible control $\mathbf{u}(\cdot) \in \mathbf{U}_{\left[t_{0}, t_{f}\right]}$ that maximizes the cost functional $J[\mathbf{u}(\cdot)]$. Suppose that the optimal control problem is solvable and $\left(\mathbf{x}^{*}, \mathbf{u}^{*}\right)$ is the optimal state-control trajectory . Then $\mathbf{u}^{*}$ and $\mathbf{x}^{*}$ can be characterized by the Pontryagin maximum principle [22].

Theorem 4 (Pontryagin maximum principle) Consider the optimal control problem (13)(17), where $U_{m} \subset \mathbf{R}^{m}$ is a closed bounded set. Suppose that

- $\mathbf{f}(\mathbf{x}, \mathbf{u}, t), \mathbf{f}_{\mathbf{x}}(\mathbf{x}, \mathbf{u}, t), L(\mathbf{x}, \mathbf{u}, t), \mathbf{g}(\mathbf{x}), \mathbf{g}_{\mathbf{x}}(\mathbf{x})$ are continuous; and

- $\mathbf{f}(\mathbf{x}, \mathbf{u}, t), \mathbf{f}_{\mathbf{x}}(\mathbf{x}, \mathbf{u}, t), L_{\mathbf{x}}(\mathbf{x}, \mathbf{u}, t)$ are bounded.

Let us introduce the Hamiltonian $H$ by

$$
H(\mathbf{x}, \mathbf{u}, \psi, t)=L(\mathbf{x}, \mathbf{u}, t)+\psi^{T} \mathbf{f}(\mathbf{x}, \mathbf{u}, t)
$$

where $\psi \in \mathbf{R}^{n}$. If $\left(\mathbf{u}^{*}, \mathbf{x}^{*}\right)$ is the optimal solution of the problem, then there exist a vectorvalued function $\psi:\left[t_{0}, t_{f}\right] \rightarrow \mathbf{R}^{n}$ and a constant vector $\mu \in \mathbf{R}^{n}$ such that $\left(\mathbf{x}^{*}(t), \psi(t)\right)$ satisfies the following Hamiltonian system:

$$
\left\{\begin{array}{l}
\dot{\mathbf{x}}^{*}(t)=\frac{\partial H}{\partial \psi^{T}}\left(\mathbf{x}^{*}(t), \mathbf{u}^{*}(t), \psi(t), t\right), \\
\dot{\psi}^{T}(t)=-\frac{\partial H}{\partial \mathbf{x}}\left(\mathbf{x}^{*}(t), \mathbf{u}^{*}(t), \psi(t), t\right),
\end{array}\right.
$$


and boundary conditions

$$
\begin{aligned}
& \mathbf{x}^{*}\left(t_{0}\right)=\mathbf{x}_{0}, \quad \mathbf{g}\left(\mathbf{x}^{*}\left(t_{f}\right)\right)=0, \\
& \psi^{T}\left(t_{f}\right)=-\mu^{T} \frac{\partial \mathbf{g}\left(\mathbf{x}^{*}\left(t_{f}\right)\right)}{\partial \mathbf{x}} .
\end{aligned}
$$

Moreover, one has

$$
H\left(\mathbf{x}^{*}(t), \mathbf{u}^{*}(t), \psi(t)\right) \equiv \text { const. } \quad \forall t \in\left[t_{0}, t_{f}\right]
$$

and, at any $t \in\left[t_{0}, t_{f}\right]$ such that $\mathbf{u}^{*}(\cdot)$ is continuous at $t$, there holds

$$
H\left(\mathbf{x}^{*}(t), \mathbf{u}^{*}(t), \psi(t)\right)=\max _{\hat{\mathbf{u}} \in U_{m}} H\left(\mathbf{x}^{*}(t), \hat{\mathbf{u}}, \psi(t)\right)
$$

For problem (1)-(2), the construction of non-degenerate potentials $q \in \Omega_{A, B}$ can be stated as the following optimal control problem. By setting $\left(x_{1}, x_{2}\right)=(x, \dot{x})$, equation (1) is equivalent to the following system:

$$
\dot{x}_{1}=x_{2}, \quad \dot{x}_{2}=-q(t) x_{1},
$$

the initial condition can be taken as

$$
\left(x_{1}(0), x_{2}(0)\right)=(0,1)
$$

The final state is

$$
x_{1}(1)=0 \text {. }
$$

The set of admissible controls is

$$
\hat{\Omega}_{A, B}:=\left\{q \in \Omega_{A, B}: \text { problem (1)-(2) has nontrivial solutions }\right\} .
$$

Finally, the cost functional on $\hat{\Omega}_{A, B}$ is

$$
J[q]=\int_{0}^{1} q(t) d t, \quad q \in \hat{\Omega}_{A, B}
$$

In the following we consider the case that $A \in\left(-\infty, \mu_{n}\right]$ is finite.

Lemma 5 The optimal control problem (24)-(27) associated with problem (1)-(2) has an optimal control potential $q^{*} \in \Omega_{A, B}$.

Proof As $q \equiv \mu_{n} \in \hat{\Omega}_{A, B}, \hat{\Omega}_{A, B} \neq \emptyset$. Define

$$
r:=\sup _{q \in \hat{\Omega}_{A, B}} J[q] \in[A, B]
$$


One has $q_{k} \in \hat{\Omega}_{A, B}$ such that $\lim _{k \rightarrow \infty} J\left[q_{k}\right]=r$. It is well known that the order-interval $\Omega_{A, B}$ is a compact subset of $L^{1}[0,1]$ when the weak topology $w_{1}$ is considered. Since $\left\{q_{k}\right\} \subset$ $\hat{\Omega}_{A, B} \subset \Omega_{A, B}$, without loss of generality, let us assume that $q_{k} \rightarrow q^{*}$ in $\left(L^{1}[0,1], w_{1}\right)$. Thus $q^{*} \in \Omega_{A, B}$.

Let $x_{k}(t), k \in \mathbf{N}$, be solutions of

$$
\ddot{x}_{k}+q_{k}(t) x_{k}=0 \quad \text { in }[0,1]
$$

satisfying $\left(x_{k}(0), \dot{x}_{k}(0)\right)=(0,1)$. By the continuity dependence results of solutions on potentials in [21], as $k \rightarrow \infty, x_{k}$ converges in $\left(C[0,1],\|\cdot\|_{C^{0}}\right)$ to $x$ which is the unique solution of

$$
\ddot{x}+q^{*}(t) x=0 \quad \text { in }[0,1]
$$

satisfying $(x(0), \dot{x}(0))=(0,1)$. Since $x_{k}(1) \equiv 0$ for all $k$, one has $x(1)=0$. Since $x \neq 0$, we see that $q^{*} \in \hat{\Omega}_{A, B}$. Finally, $q_{k} \rightarrow q^{*}$ in $\left(L^{1}[0,1], w_{1}\right)$ implies that $J\left[q_{k}\right] \rightarrow J\left[q^{*}\right]$. Hence $J\left[q^{*}\right]=r$, i.e., $q^{*}$ is an optimal control.

Lemma 6 Let $q^{*} \in \hat{\Omega}_{A, B}$ be the optimal control in Lemma 5. Define

$$
r(A, B):=\int_{[0,1]} q^{*}(t) d t \in\left[\mu_{n}, B\right) .
$$

Then any potential $q \in \Omega_{A, B}$ satisfying (8) is non-degenerate with respect to problem (1)-(2).

Proof Suppose that $q \in \Omega_{A, B}$ satisfies (8). If problem (1)-(2) has non-trivial solutions, one would have $q \in \hat{\Omega}_{A, B}$ and $J\left[q^{*}\right]=\sup _{\hat{q} \in \hat{\Omega}_{A, B}} J[\hat{q}] \geq J[q]$, a contradiction with (8).

Since $q \equiv B \in \Omega_{A, B}$ is non-degenerate, one has $q^{*} \prec B$ and $r(A, B)<B$. On the other hand, as $q \equiv \mu_{n} \in \Omega_{A, B}$ is degenerate, one has $r(A, B) \geq J\left[\mu_{n}\right]=\mu_{n}$. These have given the bounds (28) for $r(A, B)$.

\section{Construction of non-degenerate potentials for the Dirichlet problem}

In this section, we apply the PMP to find the optimal control $q^{*}$ and complete the proof of Theorem 3. To this end, we need only to take $n=2, m=k=1$. From the settings (24)-(27), let us take

$$
\begin{aligned}
& \mathbf{f}(\mathbf{x}, q, t)=\left(\begin{array}{c}
x_{2} \\
-q x_{1}
\end{array}\right), \\
& \mathbf{x}_{0}=\left(\begin{array}{l}
0 \\
1
\end{array}\right), \\
& \mathbf{g}(\mathbf{x})=x_{1}, \\
& L(\mathbf{x}, q, t)=q, \\
& U_{1}=[A, B] \subset \mathbf{R}^{1} .
\end{aligned}
$$

Then the Hamiltonian (18) in the PMP is

$$
H=q+\psi^{T} \mathbf{f}(\mathbf{x}, q, t)=q+\psi_{1} x_{2}-\psi_{2} q x_{1}, \quad \text { where } \psi \in \mathbf{R}^{2} .
$$


Let $q^{*} \in \Omega_{A, B}$ and $\left(x_{1}^{*}, x_{2}^{*}\right)$ be the optimal solutions. Then (19)-(21) in the PMP read as

$$
\begin{array}{ll}
\dot{x}_{1}^{*}=x_{2}^{*}, \quad \dot{x}_{2}^{*}=-q^{*}(t) x_{1}^{*}, \\
\dot{\psi}_{1}=q^{*}(t) \psi_{2}, \quad \dot{\psi}_{2}=-\psi_{1}, \\
\mathbf{x}^{*}(0)=\left(\begin{array}{l}
0 \\
1
\end{array}\right), \quad \mathbf{x}^{*}(1)=\left(\begin{array}{l}
0 \\
v
\end{array}\right), \\
\psi(1)=\left(\begin{array}{c}
-\mu \\
0
\end{array}\right),
\end{array}
$$

where $\mu, v \in \mathbf{R}$ are some constants.

Note that system (31) is essentially the same as (30). In fact, both $x_{1}^{*}(t)$ and $\psi_{2}(t)$ are solutions of the following equation:

$$
\ddot{y}+q^{*}(t) y=0, \quad t \in[0,1]
$$

Moreover, from (32) and (33), one has

$$
\left(x_{1}^{*}(1), \dot{x}_{1}^{*}(1)\right)=(0, v) \quad \text { and } \quad\left(\psi_{2}(1), \dot{\psi}_{2}(1)\right)=(0, \mu) .
$$

Thus there exists a constant $c$ such that

$$
\psi_{2}(t) \equiv c x_{1}^{*}(t) \quad \text { and } \quad \psi_{1}(t) \equiv-c x_{2}^{*}(t), \quad t \in[0,1]
$$

Recall that eigenvalues $\lambda_{m}(q)$ of problem (3)-(2) have the following comparison properties:

$$
q_{i} \in L^{1}[0,1], \quad q_{1} \leq q_{2} \quad \Longrightarrow \quad \lambda_{m}\left(q_{1}\right) \geq \lambda_{m}\left(q_{2}\right) \quad \text { for all } m \in \mathbf{N},
$$

and

$$
q_{i} \in L^{1}[0,1], \quad q_{1} \prec q_{2} \quad \Longrightarrow \quad \lambda_{m}\left(q_{1}\right)>\lambda_{m}\left(q_{2}\right) \quad \text { for all } m \in \mathbf{N},
$$

where $q_{1} \prec q_{2}$ means that $q_{1} \leq q_{2}$ and $q_{1}(t)<q_{2}(t)$ on some subset of $[0,1]$ of positive measure. It is trivial that

$$
\lambda_{m}(q+\gamma)=\lambda_{m}(q)-\gamma
$$

for any constant $\gamma$, we see that condition $B \in\left(\mu_{n}, \mu_{n+1}\right)$ is equivalent to

$$
\lambda_{n}(B)<0<\lambda_{n+1}(B)
$$

As for the optimal potential $q^{*}$ in Lemma 5, one has the following results.

Lemma 7 The optimal control potential $q^{*}$ in Lemma 5 must satisfy $\lambda_{n}\left(q^{*}\right)=0$. Consequently, as an eigenfunction, $x_{1}^{*}(t)$ has precisely $(n+1)$ zeros in $[0,1]$, say $0=t_{0}<t_{1}<\cdots<$ $t_{n}=1$. 
Proof Since $q^{*} \in \hat{\Omega}_{A, B}$, one has $\lambda_{m}\left(q^{*}\right)=0$ for some $m \in \mathbf{N}$. As $q^{*} \leq B$, by the comparison results for eigenvalues, one has $\lambda_{n+1}\left(q^{*}\right) \geq \lambda_{n+1}(B)>0$. Thus $m \leq n$.

We assert that $m=n$ and hence one has $\lambda_{n}\left(q^{*}\right)=0$. Otherwise, assume that $m \leq n-1$. Denote

$$
q_{\tau}:=(1-\tau) q^{*}+\tau B \in \Omega_{A, B}, \quad \tau \in[0,1]
$$

One has $\lambda_{n}\left(q_{0}\right)=\lambda_{n}\left(q^{*}\right)>\lambda_{m}\left(q^{*}\right)=0$ and $\lambda_{n}\left(q_{1}\right)=\lambda_{n}(B)<0$. By the continuity of $\lambda_{n}\left(q_{\tau}\right)$ in $\tau$, there exists $\tau_{0} \in(0,1)$ such that $\lambda_{n}\left(q_{\tau_{0}}\right)=0$. Since $q_{\tau_{0}} \in \hat{\Omega}_{A, B}$ and

$$
\int_{[0,1]} q_{\tau_{0}}(t) d t>\int_{[0,1]} q^{*}(t) d t
$$

it is a contradiction with the optimality of $q^{*}$.

In order to deduce the optimal potential $q^{*}$, we will use properties (22) and (23) in the PMP. For simplicity, we write

$$
y(t):=x_{1}^{*}(t), \quad t \in[0,1]
$$

which is a solution of problem (34)-(2) and satisfies $(y(0), \dot{y}(0))=(0,1)$.

By (29) and (35), $H$ takes the following form:

$$
H\left(\mathbf{x}^{*}(t), q, \psi(t)\right)=q+\psi_{1}(t) x_{2}^{*}(t)-q \psi_{2}(t) x_{1}^{*}(t) \equiv\left(1-c y^{2}(t)\right) q-c \dot{y}^{2}(t)=: G(t, q)
$$

where $q \in[A, B]$. When $t$ is fixed, as a function of $q \in[A, B], G(t, q)$ attains its maximum at

$$
q= \begin{cases}B & \text { if } 1-c y^{2}(t)>0, \\ A & \text { if } 1-c y^{2}(t)<0 \\ \in[A, B] & \text { if } 1-c y^{2}(t)=0 .\end{cases}
$$

From (23), we conclude that, at any $t \in[0,1]$ such that $q^{*}(\cdot)$ is continuous at $t$, there holds

$$
q^{*}(t)= \begin{cases}B & \text { if } 1-c y^{2}(t)>0, \\ A & \text { if } 1-c y^{2}(t)<0, \\ \in[A, B] & \text { if } 1-c y^{2}(t)=0 .\end{cases}
$$

If $c \leq 0$, we would have $q^{*}=B$, which is impossible. Hence $c>0$. Let us denote $c_{*}:=1 / \sqrt{c}>0$. Then, at any $t \in[0,1]$ such that $q^{*}(\cdot)$ is continuous at $t$, one has

$$
q^{*}(t)= \begin{cases}B & \text { if }|y(t)|<c_{*}, \\ A & \text { if }|y(t)|>c_{*}, \\ \in[A, B] & \text { if }|y(t)|=c_{*} .\end{cases}
$$

Moreover, from (22), one has

$$
H=\left(1-y^{2}(t) / c_{*}^{2}\right) q^{*}(t)-\dot{y}^{2}(t) / c_{*}^{2} \equiv \text { const. }
$$


For each $i=0,1, \ldots, n$, as $y\left(t_{i}\right)=0$, one has $|y(t)|<c_{*}$ on some (maximal) interval $I_{i}$ containing $t_{i}$. By (37), $q^{*}(t)=B$ on $I_{i}$. Thus (38) implies that $B-\dot{y}^{2}\left(t_{i}\right) / c_{*}^{2} \equiv$ const. Hence $\dot{y}\left(t_{i+1}\right)=-\dot{y}\left(t_{i}\right)$. Since $\dot{y}\left(t_{0}\right)$ is assumed to be 1 , we conclude

$$
\left(y\left(t_{i}\right), \dot{y}\left(t_{i}\right)\right)=\left(0,(-1)^{i}\right), \quad i=0,1, \ldots, n .
$$

Moreover, on $I_{i}, y(t)$ satisfies

$$
\ddot{y}+B y=0 .
$$

Using conditions (39), we know that, on $I_{i}, y(t)$ is given by

$$
y(t)=(-1)^{i} \frac{\sin \left(\sqrt{B}\left(t-t_{i}\right)\right)}{\sqrt{B}} .
$$

Let us introduce

$$
J:=\left\{t \in(0,1):|y(t)| \geq c_{*}\right\}
$$

Then $J$ is a closed set contained in $(0,1)$. Note that $q^{*}(t)=B$ on $[0,1] \bigvee$ and $q^{*} \neq B$. Thus $J \neq \emptyset$ and $J$ consists of closed intervals

$$
J=\bigcup J_{j}
$$

where $J_{j}=\left[\xi_{j}, \eta_{j}\right] \subset(0,1)$ may shrink into a single point. However, as $q^{*} \neq B$, at least one of $J_{j}$ 's is a non-trivial closed interval.

Lemma 8 The number of intervals $J_{j}$ 's in (41), including the degenerate ones, is precisely $n$. Moreover, by labeling $J_{i}$ according to the order in $\mathbf{R}$, one has $J_{i} \subset\left(t_{i-1}, t_{i}\right)$ for $i=1,2, \ldots, n$, where $t_{i}$ 's are as in Lemma 7.

Proof Note that $t_{i} \notin J$ for all $i=0,1, \ldots, n$. Thus

$$
J \subset \bigcup_{i=1}^{n}\left(t_{i-1}, t_{i}\right) .
$$

Step 1. For each $i=1,2, \ldots, n$, we assert that $\left(t_{i-1}, t_{i}\right)$ contains at most one interval $J_{j}$ from (41).

Otherwise, we would have two neighboring intervals $[\xi, \eta]$ and $[\hat{\xi}, \hat{\eta}]$ from $J_{j}$ 's such that $t_{i-1}<\xi \leq \eta<\hat{\xi} \leq \hat{\eta}<t_{i}$ and $|y(t)|<c_{*}$ on $(\eta, \hat{\xi})$. Since $y(t)$ does not change sign in $\left(t_{i-1}, t_{i}\right)$, let us assume that $y(\eta)=y(\hat{\xi})=c_{*}$ and $0<y(t)<c_{*}$ on $(\eta, \hat{\xi})$. Then, on $(\eta, \hat{\xi})$, $\ddot{y}(t)=-q^{*}(t) y(t)=-B y(t)<0$ and $y(t)$ is strictly concave. Hence we would have $y(t)>c_{*}$ on $(\eta, \hat{\xi})$, a contradiction.

Step 2. Let $J_{j}=[\xi, \eta]$ be any interval from (41) such that $J_{j}=[\xi, \eta] \subset\left(t_{i-1}, t_{i}\right)$. For $t \in\left[\eta, t_{i}\right]$, $y(t)$ is given by (40). Thus $c_{*}=|y(\eta)| \leq 1 / \sqrt{B}$.

Step 3 . For each $i=1,2, \ldots, n$, we assert that $\left(t_{i-1}, t_{i}\right)$ contains precisely one interval $J_{j}$ from (41). 
Otherwise, from Step 1, let us assume that $\left(t_{i-1}, t_{i}\right)$ contains no $J_{j}$ from (41). Thus $|y(t)|<$ $c_{*}$ on $\left(t_{i-1}, t_{i}\right)$. On $\left[t_{i-1}, t_{i}\right], y(t)$ is given by (40). As $y\left(t_{i-1}\right)=0$, we have from (40) that $t_{i}-$ $t_{i-1}=\pi / \sqrt{B}$. Thus $c_{*}>\left|y\left(\left(t_{i-1}+t_{i}\right) / 2\right)\right|=1 / \sqrt{B}$, a contradiction with the result on $c_{*}$ in Step 2.

For $i=1,2, \ldots, n$, denote $J_{i}=\left[\xi_{i}, \eta_{i}\right] \subset\left(t_{i-1}, t_{i}\right)$. We have obtained the following formulas:

$$
\begin{aligned}
& q^{*}(t)=B \quad \text { for } t \in\left[t_{i-1}, \xi_{i}\right] \cup\left[\eta_{i}, t_{i}\right], \\
& y(t)= \begin{cases}(-1)^{i-1} \frac{\sin \left(\sqrt{B}\left(t-t_{i-1}\right)\right)}{\sqrt{B}} & \text { for } t \in\left[t_{i-1}, \xi_{i}\right], \\
(-1)^{i} \frac{\sin \left(\sqrt{B}\left(t-t_{i}\right)\right)}{\sqrt{B}} & \text { for } t \in\left[\eta_{i}, t_{i}\right] .\end{cases}
\end{aligned}
$$

These give $q^{*}(t)$ and $y(t)$ on $[0,1] \bigvee$. Moreover, as $\left|y\left(\xi_{i}\right)\right|=\left|y\left(\eta_{i}\right)\right|=c_{*}$, we have from (43)

$$
\xi_{i}-t_{i-1}=t_{i}-\eta_{i} \equiv \tau, \quad i=1,2, \ldots, n,
$$

where $\tau=\arcsin \left(c_{*} \sqrt{B}\right) / \sqrt{B}>0$ is a constant. Note that $\tau \leq \pi / 2 \sqrt{B}$.

In order to construct $q^{*}(t)$ and $y(t)$ on $J$, we need to distinguish two cases.

Case $0<A \leq \mu_{n}$. Let us first consider a non-trivial interval from $J_{i}$ 's, say $J_{1}=\left[\xi_{1}, \eta_{1}\right]$, where $\xi_{1}<\eta_{1}$. Since $q^{*} \geq A>0$, equation (34) implies that $y(t)$ is strictly concave on $\left[t_{0}, t_{1}\right]$. Thus $y(t)>c_{*}=y\left(\xi_{1}\right)=y\left(\eta_{1}\right)$ on $\left(\xi_{1}, \eta_{1}\right)$. By (37), one has

$$
q^{*}(t)=A, \quad t \in\left(\xi_{1}, \eta_{1}\right)
$$

Since $y(t)$ satisfies

$$
\ddot{y}+A y=0
$$

on $\left(\xi_{1}, \eta_{1}\right), y(t)$ is symmetric with respect to $t=\left(\xi_{1}+\eta_{1}\right) / 2$ and

$$
y(t)=C \cos \left(\sqrt{A}\left(t-\left(\xi_{1}+\eta_{1}\right) / 2\right)\right), \quad t \in\left(\xi_{1}, \eta_{1}\right),
$$

where $C \neq 0$ is some constant. In order for $y(t)$ to be $C^{1}$ on $\left[t_{0}, t_{1}\right]$, it is necessary and sufficient that

$$
\left(y\left(\xi_{1}+\right), \dot{y}\left(\xi_{1}+\right)\right)=\left(y\left(\xi_{1}-\right), \dot{y}\left(\xi_{1}-\right)\right) .
$$

By formulas (43) and (46), this is

$$
\sqrt{B} \cot (\sqrt{B} \tau)=\sqrt{A} \tan \left(\sqrt{A} \frac{\left|J_{1}\right|}{2}\right)
$$

where $\tau$ is as in (44). Hence the length $\left|J_{1}\right|=\eta_{1}-\xi_{1}$ is uniquely determined by the parameter $\tau$.

Next, for any $i$, it follows from (43) and (44) that $J_{i}=\left[\xi_{i}, \eta_{i}\right]$ has the same length with $\left|J_{1}\right|$. Hence $t_{i}-t_{i-1}=1 / n$ for all $i$, i.e., $t_{i}=i / n$ for $i=0,1, \ldots, n$. By using the parameter $\tau$ in (44), one has then $\left|J_{i}\right|=1 / n-2 \tau$. 
Finally, let us introduce the following parameter:

$$
\alpha:=2 n \tau \in(0, n \pi / \sqrt{B}] \subset(0,1) .
$$

Then $\left|J_{i}\right|=1 / n-2 \tau=(1-\alpha) / n$ and equation (47) reads as

$$
\sqrt{B} \cot \frac{\alpha \sqrt{B}}{2 n}=\sqrt{A} \tan \frac{(1-\alpha) \sqrt{A}}{2 n} .
$$

When $A$ and $B$ are fixed, it is easy to verify that equation (49) has a unique solution $\alpha=\alpha(A, B)$ in the interval (48) for $\alpha$. In fact, one has $0<\alpha(A, B)<n \pi / \sqrt{B}$ in this case. Moreover, $q^{*}(t)$ is determined by (42) and (45).

Lemma 9 Suppose that $B$ is as in (6) and $A \in\left(0, \mu_{n}\right]$. Then the optimal control potential $q^{*} \in \Omega_{A, B}$ is unique. Moreover, one has $q^{*}(t) \equiv q^{*}(t-(i-1) / n)$ for all $t \in[(i-1) / n, i / n]$, where $i=1,2, \ldots, n$, and $\left.q^{*}\right|_{[0,1 / n]}$ is given by

$$
q^{*}(t)= \begin{cases}B & \text { for } t \in[0, \alpha / 2 n] \cup[1 / n-\alpha / 2 n, 1 / n], \\ A & \text { for } t \in(\alpha / 2 n, 1 / n-\alpha / 2 n),\end{cases}
$$

where $\alpha=\alpha(A, B) \in(0, n \pi / \sqrt{B})$ is the unique solution of equation (49).

Case $A \leq 0$. Denote $J_{i}=\left[\xi_{i}, \eta_{i}\right] \subset\left(t_{i-1}, t_{i}\right)$. At first, we assert that $y(t) \equiv(-1)^{i+1} c_{*}$ on $J_{i}$. This is trivial when $\xi_{i}=\eta_{i}$. In the following, we assume that $\xi_{i}<\eta_{i}$. If $y(t)$ is not constant on $J_{i}$, one would have a non-trivial subinterval $(\xi, \eta) \subset\left[\xi_{i}, \eta_{i}\right]$ such that

$$
(-1)^{i+1} y(t)>c_{*} \quad \text { on }(\xi, \eta), \quad \text { and } \quad(-1)^{i+1} y(\xi)=(-1)^{i+1} y(\eta)=c_{*} .
$$

Then, on $(\xi, \eta)$, it follows from (37) that $q^{*}(t)=A \leq 0$ and from equation (34) that $(-1)^{i+1} \ddot{y}(t)=-A(-1)^{i+1} y(t) \geq 0$. Thus $(-1)^{i+1} \dot{y}(t)$ is non-decreasing. However, (51) implies that $(-1)^{i+1} \dot{y}(\xi) \geq 0$ and $(-1)^{i+1} \dot{y}(\eta) \leq 0$. Therefore $(-1)^{i+1} \dot{y}(t) \equiv 0$ and $y(t)$ is constant on $\left(\xi_{i}, \eta_{i}\right)$, a contradiction with assumption (51).

Next we assert that

$$
\dot{y}(t)=0 \quad \forall t \in J_{i}=\left[\xi_{i}, \eta_{i}\right] .
$$

In fact, for any $t \in J_{i}$, one has $(-1)^{i+1} y(t)=\max _{s \in\left(t_{i-1}, t_{i}\right)}(-1)^{i+1} y(s)$. Hence one has (52).

Finally, from (43), (44) and (52), one knows that $\tau$ satisfies $\cos (\sqrt{B} \tau)=0$. Hence

$$
\tau=\pi / 2 \sqrt{B}
$$

Moreover, as $(-1)^{i+1} y(t) \equiv c_{*}$ on $\left(\xi_{i}, \eta_{i}\right)$, we obtain from equation (34) that

$$
q^{*}(t)=0, \quad t \in\left(\xi_{i}, \eta_{i}\right), i=1,2, \ldots, n
$$

Lemma 10 Suppose that $B$ is as in (6) and $A \in(-\infty, 0]$. Then the optimal control potential $q^{*} \in \Omega_{A, B}$ is not unique. By letting

$$
\alpha=\alpha(A, B):=2 n \tau=n \pi / \sqrt{B} \in(0,1),
$$


one optimal control potential $q^{*}$ can be chosen so that $q^{*}(t) \equiv q^{*}(t-(i-1) / n)$ for all $t \in$ $[(i-1) / n, i / n]$, where $i=1,2, \ldots, n$, and $\left.q^{*}\right|_{[0,1 / n]}$ is given by

$$
q^{*}(t)= \begin{cases}B & \text { for } t \in[0, \alpha / 2 n] \cup[1 / n-\alpha / 2 n, 1 / n], \\ 0 & \text { for } t \in(\alpha / 2 n, 1 / n-\alpha / 2 n) .\end{cases}
$$

Here formulas (55) and (56) are deduced from (53) and from (42), (54), respectively.

Proof of Theorem 3 Case 1: $A \in\left(0, \mu_{n}\right]$. From Lemma 9 and formula (50), one has

$$
r(A, B)=\int_{0}^{1} q^{*}(t) d t=n \int_{0}^{1 / n} q^{*}(t) d t=(1-\alpha) A+\alpha B,
$$

where $\alpha=\alpha(A, B)$ is the solution of equation (49). By letting $r=r(A, B)$, one has from (57) that $\alpha=(r-A) /(B-A)$ and $1-\alpha=(B-r) /(B-A)$. Substituting into (49), we know that $r=r(A, B)$ satisfies equation (10). Moreover, one has from Lemma 6 that $r \geq \mu_{n}$ and from (48) that $r=A+\alpha(B-A)<A+n \pi(B-A) / \sqrt{B}$. Hence $r$ is inside the interval (9) in this case.

Case 2: $A \in(-\infty, 0]$. From (55) and (56), one has

$$
r(A, B)=n \int_{0}^{1 / n} q^{*}(t) d t=n B(\alpha / n)=n \pi \sqrt{B} .
$$

This is (11).

In these two cases, denote

$$
\check{\Omega}_{A, B}:=\left\{q \in L^{1}[0,1]: A \leq q(t) \leq B \text { a.e. } t \in[0,1] \text {, and } \int_{0}^{1} q(t) d t>r(A, B)\right\} .
$$

Lemma 6 asserts that any $q \in \check{\Omega}_{A, B}$ is non-degenerate. In fact, as $\check{\Omega}_{A, B}$ is a convex set containing $B$, for any $q \in \check{\Omega}_{A, B}$, one has $(1-s) q+s B \in \check{\Omega}_{A, B}$ for $s \in[0,1]$. By Lemma 2,

$$
\Lambda_{m}(s):=\lambda_{m}((1-s) q+s B) \neq 0 \quad \forall s \in[0,1], \forall m \in \mathbf{N} .
$$

As continuous functions of $s$, one has then

$$
0<\Lambda_{m}(0) \Lambda_{m}(1)=\lambda_{m}(q) \lambda_{m}(B) \quad \forall m \in \mathbf{N}
$$

Thus $\lambda_{n}(q)$ and $\lambda_{n+1}(q)$ have the same signs as those of $\lambda_{n}(B)$ and $\lambda_{n+1}(B)$, respectively. Due to (36), we obtain (12).

Moreover, the optimality of $r(A, B)$ follows from Lemmas 5 and 6 , by simply taking $\hat{q}=q^{*}$.

Case 3: $A=-\infty$. In this case, one has $\Omega_{-\infty, B} \supset \Omega_{A, B}$ for any $A \in(-\infty, 0]$. By the meaning of $r(-\infty, B)$, one has $r(-\infty, B) \geq r(0, B)=n \pi \sqrt{B}$.

On the other hand, suppose that $q \in \Omega_{-\infty, B}$ satisfies

$$
\ell:=\int_{0}^{1} q(t) d t>n \pi \sqrt{B}
$$


Since $q \leq B$, one has

$$
\lambda_{n+1}(q) \geq \lambda_{n+1}(B)>0
$$

For any $A \in(-\infty, 0)$, define

$$
\begin{aligned}
& E_{A}:=\{t \in[0,1]: q(t)<A\}, \\
& q_{A}(t):= \begin{cases}q(t) & \text { for } t \in[0,1] \backslash E_{A}, \\
0 & \text { for } t \in E_{A} .\end{cases}
\end{aligned}
$$

Then $q_{A} \geq q$ and $q_{A} \in \Omega_{A, B}$. Let $0<\varepsilon<\ell-n \pi \sqrt{B}$ be a constant. See (58). Then $q_{A}-\varepsilon \in$ $\Omega_{A-\varepsilon, B}$ and

$$
\int_{0}^{1}\left(q_{A}(t)-\varepsilon\right) d t \geq \int_{0}^{1} q(t) d t-\varepsilon=\ell-\varepsilon>n \pi \sqrt{B} .
$$

Thus $q_{A}-\varepsilon \in \check{\Omega}_{A-\varepsilon, B}$. From inequality (12), for $q_{A}-\varepsilon$, we know that

$$
\lambda_{n}\left(q_{A}-\varepsilon\right)=\lambda_{n}\left(q_{A}\right)+\varepsilon<0
$$

When $A \downarrow-\infty$, the measure of $E_{A}$ tends to 0 and $\left\|q_{A}-q\right\|_{1} \rightarrow 0$. Therefore

$$
\lambda_{n}(q)=\lim _{A \downarrow-\infty} \lambda_{n}\left(q_{A}\right) \leq-\varepsilon<0 .
$$

See (60). Combining with (59), we know that $q$ also satisfies (12). Thus $r(-\infty, B) \leq$ $n \pi \sqrt{B}$.

Remark 11 Let $B$ be as in (6). For the case $A=-\infty$, Theorem 3 means that any potential in

$$
\begin{aligned}
\check{\Omega}_{-\infty, B}:= & \left\{q \in L^{1}[0,1]: q(t) \leq B \text { a.e. } t \in[0,1],\right. \text { and } \\
& \left.\int_{0}^{1} q(t) d t>r(-\infty, B)=n \pi \sqrt{B}\right\}
\end{aligned}
$$

is non-degenerate. Note that these non-degenerate potentials $q(t)$ may be unbounded from below.

Let us fix $B$ as in (6) and consider $r(A, B)$ as a function of $A \in\left(-\infty, \mu_{n}\right]$. From equation (10), it is easy to prove that $r=r(A, B)$ is continuous and non-increasing in $A$. Moreover, one has $r\left(\mu_{n}, B\right)=\mu_{n}=(n \pi)^{2}$. As a function of $A$, the graph of $r(A, B)$ is as in Figures 1 and 2 .

\section{Non-degenerate potentials for the Neumann problem}

For equation (1) with the Neumann boundary condition

$$
\dot{x}(0)=\dot{x}(1)=0,
$$



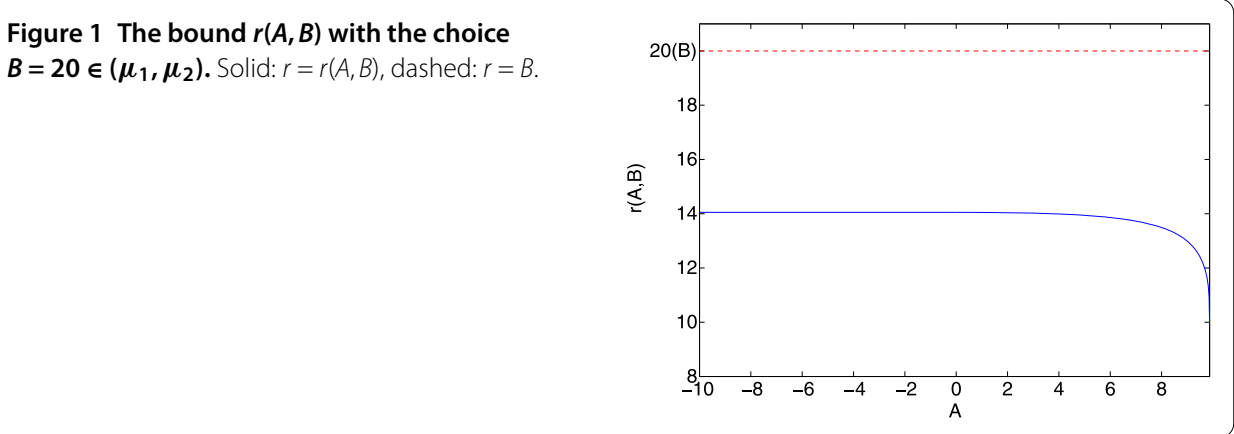

\section{Figure 2 The bound $r(A, B)$ with the choice}

$\boldsymbol{B}=\mathbf{7 0} \in\left(\boldsymbol{\mu}_{2}, \boldsymbol{\mu}_{3}\right)$. Solid: $r=r(A, B)$, dashed: $r=B$.

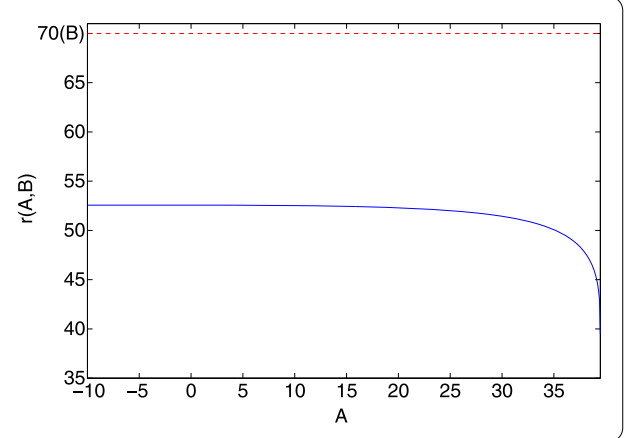

the non-degenerate potentials can be defined similarly as in Definition 1. Given a potential $q \in L^{1}[0,1]$. The eigenvalues of problem (3)-(61) are

$$
\hat{\lambda}_{0}(q)<\hat{\lambda}_{1}(q)<\cdots<\hat{\lambda}_{m}(q)<\cdots, \quad \lim _{m \rightarrow+\infty} \hat{\lambda}_{m}(q)=+\infty
$$

Note that

$$
\hat{\mu}_{m}:=\hat{\lambda}_{m}(0)=(m \pi)^{2}, \quad m \in \mathbf{Z}^{+}:=\{0\} \cup \mathbf{N} .
$$

One has $\hat{\mu}_{m}=\mu_{m}$ for $m \in \mathbf{N}$.

By the approach in the preceding sections, for the Neumann problem, the results are as follows.

Theorem 12 Let $B$ and $A$ be as in (6) and (7), where $n \in \mathbf{N}$. By letting $r(A, B)$ and $\check{\Omega}_{A, B}$ be as those for the Dirichlet problem, any $q \in \check{\Omega}_{A, B}$ is also non-degenerate with respect to problem (1)-(61).

For the Neumann problem, one has the zeroth eigenvalue. See (62) and (63). This leads to the following problem. Suppose that $B$ and $A$ are such that

$$
0<B<\mu_{1}, \quad A \leq 0 .
$$

What is the optimal lower bound $r(A, B)$ for $\int_{0}^{1} q(t) d t$ so that any $q \in \Omega_{A, B}$ is nondegenerate? 
Theorem 13 Let $B$ and $A$ be as in (64). Then $r(A, B) \equiv 0$. That is, any $q \in \Omega_{A, B}$ satisfying

$$
\int_{0}^{1} q(t) d t>0
$$

is a non-degenerate potential of problem (1)-(61). Moreover, the lower bound 0 in (65) is optimal.

Proof This theorem is simply a restatement of some classical results. In fact, for any $q \in$ $L^{1}[0,1]$, it is well known that

$$
\hat{\lambda}_{0}(q) \leq-\int_{0}^{1} q(t) d t
$$

See [17]. Under assumption (65), one has $\hat{\lambda}_{0}(q)<0$. On the other hand, for any $q \in \Omega_{A, B}$, as $q \leq B$, one has $\hat{\lambda}_{1}(q) \geq \hat{\lambda}_{1}(B)=\mu_{1}-B>0$. See assumption (64). Thus $q$ is a non-degenerate potential of problem (1)-(61).

As for the optimality, one needs only to notice that the zero potential $q=0 \in \Omega_{A, B}$ is degenerate with respect to problem (1)-(61).

\section{Competing interests}

The authors declare that they have no competing interests.

Authors' contributions

Both authors have equally contributed to this article and also read and approved the final manuscript.

\section{Acknowledgements}

The authors would like to thank Zhiyuan Wen for helpful discussions. This work is supported by the National Natural Science Foundation of China (Grant No. 11231001 and No. 11371213) and the National 111 Project of China (Station No. 111-2-01).

Received: 19 March 2015 Accepted: 6 October 2015 Published online: 16 October 2015

\section{References}

1. Chen, H, Li, Y: Stability and exact multiplicity of periodic solutions of Duffing equations with cubic nonlinearities. Proc. Am. Math. Soc. 135, 3925-3932 (2007)

2. Chen, H, Li, Y: Bifurcation and stability of periodic solutions of Duffing equations. Nonlinearity 21, 2485-2503 (2008)

3. Li, Y, Wang, H: Neumann problems for second order ordinary differential equations across resonance. Z. Angew. Math. Phys. 46, 393-406 (1995)

4. Lin, Y, Li, Y, Zhou, Q: Second boundary value problems for nonlinear ordinary differential equations across resonance. Nonlinear Anal. 28, 999-1009 (1997)

5. Meng, G, Yan, P, Lin, X, Zhang, M: Non-degeneracy and periodic solutions of semilinear differential equations with deviation. Adv. Nonlinear Stud. 6, 563-590 (2006)

6. Wang, H, Li, Y: Two point boundary value problems for second order ODEs across many resonant points. J. Math. Anal. Appl. 179, 61-75 (1993)

7. Wang, $\mathrm{H}, \mathrm{Li}, \mathrm{Y}$ : Existence and uniqueness of solutions to two point boundary value problems for ordinary differential equations. Z. Angew. Math. Phys. 47, 373-384 (1996)

8. Yang, X: Sturm-Liouville problems for second order ordinary differential equations across resonance. J. Optim. Theory Appl. 152, 814-822 (2012)

9. Cabada, A, Cid, JA: On comparison principles for the periodic Hill's equation. J. Lond. Math. Soc. (2) 86, 272-290 (2012)

10. Cabada, A, Cid, JA, Tvrdy, M: A generalized anti-maximum principle for the periodic one dimensional $p$-Laplacian with sign changing potential. Nonlinear Anal. 72, 3436-3446 (2010)

11. Torres, PJ, Zhang, M: A monotone iterative scheme for a nonlinear second order equation based on a generalized anti-maximum principle. Math. Nachr. 251, 101-107 (2003)

12. Zhang, M: Optimal conditions for maximum and antimaximum principles of the periodic solution problem. Bound. Value Probl. 2010, Article ID 410986 (2010)

13. Kunze, M, Ortega, R: On the number of solutions to semilinear boundary value problems. Adv. Nonlinear Stud. 4, 237-249 (2004)

14. Li, W, Zhang, M: Non-degeneracy and uniqueness of periodic solutions for some superlinear beam equations. Appl. Math. Lett. 22, 314-319 (2009)

15. Ortega, R, Zhang, M: Optimal bounds for bifurcation values of a superlinear periodic problem. Proc. R. Soc. Edinb., Sect. A, Math. 135, 119-132 (2005) 
16. Torres, PJ, Cheng, Z, Ren, J: Non-degeneracy and uniqueness of periodic solutions for $2 n$-order differential equations. Discrete Contin. Dyn. Syst., Ser. A 33, 2155-2168 (2013)

17. Zhang, M: Certain classes of potentials for $p$-Laplacian to be non-degenerate. Math. Nachr. 278, 1823-1836 (2005)

18. Cañada, A, Montero, JA, Villegas, S: Lyapunov-type inequalities and Neumann boundary value problems at resonance. Math. Inequal. Appl. 8, 459-475 (2005)

19. Cañada, A, Montero, JA, Villegas, S: Lyapunov inequalities for partial differential equations. J. Funct. Anal. 237, 176-193 (2006)

20. Cañada, A, Villegas, S: Lyapunov inequalities for Neumann boundary conditions at higher eigenvalues. J. Eur. Math. Soc. 12, 163-178 (2010)

21. Zhang, M: Continuity in weak topology: higher order linear systems of ODE. Sci. China Ser. A 51, 1036-1058 (2008)

22. Athans, M, Falb, PL: Optimal Control: An Introduction to the Theory and Its Applications. McGraw-Hill, New York (1966)

Submit your manuscript to a SpringerOpen ${ }^{\circ}$ journal and benefit from:

- Convenient online submission

- Rigorous peer review

- Immediate publication on acceptance

- Open access: articles freely available online

- High visibility within the field

- Retaining the copyright to your article 